\title{
A. Übersicht über die Finanz- und Handelsströme zwischen der Schweiz und den Entwicklungsländern
}

Gérard Perroulaz, Xavier Tschumi Canosa and Tanja Guggenbühl

\section{OpenEdition}

\section{Journals}

Electronic version

URL: http://journals.openedition.org/sjep/89

DOI: $10.4000 /$ sjep.89

ISSN: 1663-9677

Publisher

Institut de hautes études internationales et du développement

\section{Printed version}

Date of publication: 1 avril 2008

Number of pages: $231-240$

ISBN: 978-2-940415-02-1

ISSN: $1660-5926$

\section{Electronic reference}

Gérard Perroulaz, Xavier Tschumi Canosa und Tanja Guggenbühl, « A. Übersicht über die Finanz- und Handelsströme zwischen der Schweiz und den Entwicklungsländern », Schweizerisches Jahrbuch für Entwicklungspolitik [Online], 27-1 | 2008, Online erschienen am: 21 Juni 2010, abgerufen am 08

September 2020. URL : http://journals.openedition.org/sjep/89 ; DOI : https://doi.org/10.4000/sjep.89 


\section{A. Übersicht über die Finanz- und Handelsströme zwischen der Schweiz und den Entwicklungs- ländern}

\section{A.1. Kommentar zu den Zahlen der Tabellen A}

Tabelle A.1 vermittelt für die Schweiz eine nach Ländergruppen aufgeteilte Übersicht über die öffentliche Entwicklungshilfe (ÖEH) und andere Beiträge des öffentlichen Sektors, die private Hilfe der Nichtregierungsorganisationen (NRO), die im Rahmen der Exportrisikogarantie (ERG) gezahlten Beträge, die ausländischen Direktinvestitionen (ADI) sowie die Einfuhren und Ausfuhren von Gütern im Jahr 2006. Diese Übersicht umfasst nur die Empfängerländer öffentlicher Entwicklungshilfe gemäss der Liste des OECD-Entwicklungshilfeausschusses (DAC). Die Liste ist am Schluss des Jahrbuchs aufgeführt.

Die am wenigsten entwickelten Länder (LDC) haben im Jahr 2006 zwar ein Fünftel der bilateralen öffentlichen Entwicklungshilfe der Schweiz und praktisch zwei Fünftel der privaten Hilfe der NRO erhalten, jedoch fliessen nur sehr wenig ausländische Direktinvestitionen (ADI) der Schweiz in diese Länder, die auch nur einen geringen Prozentsatz des Schweizer Aussenhandels mit den Empfängerländern öffentlicher Entwicklungshilfe ausmachen.

Auf der DAC-Liste werden die Empfängerländer öffentlicher Entwicklungshilfe - neben den am wenigsten entwickelten Ländern (LDC) - in einkommensschwache Länder (mit unter 825 US-Dollar pro Einwohner für 2004) und in Länder mit mittlerem Einkommen (zwischen 826 und 3255 US-Dollar pro Einwohner 2004 für die untere Einkommensstufe und zwischen 3256 und 10065 US-Dollar pro Einwohner 2004 für die obere Einkommensstufe) aufgeteilt. Diese Länder erhalten natürlich einen Teil der öffentlichen Entwicklungshilfe der Schweiz und der privaten Hilfe der NRO, hingegen fliesst ihnen fast die Gesamtheit der für die Empfängerländer öffentlicher Entwicklungshilfe bestimmten Schweizer Direktinvestitionen im Ausland zu. Betreffend den Aussenhandel der Schweiz mit diesen Ländern für 2006 entfallen mehr als vier von fünf Franken auf die Länder mit mittlerem Einkommen, sowohl für die Einfuhren als auch für die Ausfuhren.

In absoluten Zahlen ist es interessant, festzustellen, dass der Betrag der öffentlichen Entwicklungshilfe und anderer Beiträge des öffentlichen Sektors in die Empfängerländer öffentlicher Entwicklungshilfe im Jahr 2006 fast achtmal geringer ist als der Betrag der ausländischen Direktinvestitionen der Schweiz in diese Länder. Für die am wenigsten entwickelten Länder (LDC) sind die öffentlichen Entwicklungshilfeflüsse und andere Beiträge des öffentlichen Sektors achtmal höher als die ausländischen Direktinvestitionen, hingegen ist bei den anderen Empfängerländern (ohne LDC) genau das Gegenteil der Fall: Bei diesen Ländern machen die öffentliche Entwicklungshilfe und andere öffentliche Beiträge weniger als ein Zwanzigstel der ausländischen Direktinvestitionen aus. 
Der Aussenhandel der Schweiz mit den Empfängerländern öffentlicher Entwicklungshilfe weist für die Gesamtheit dieser Länder einen positiven Handelssaldo aus. Dies gilt aber auch für jede einzelne der vom DAC festgelegten Länderkategorien (am wenigsten entwickelte Länder, einkommensschwache Länder und Länder mit mittlerem Einkommen). Unter ,positiver Handelssaldo“ ist zu verstehen, dass der Betrag der Ausfuhren der Schweiz in diese Länder höher ist als der Betrag ihrer Einfuhren aus diesen Ländern. Tabelle A.2 zeigt, dass Afrika der einzige Kontinent ist, mit dem die Schweiz einen negativen Handelssaldo verzeichnet. Dies ist hauptsächlich auf den hohen Betrag der Erdölimporte aus Libyen und Nigeria zurückzuführen.

In Tabelle A.3 sind noch einige Zahlen betreffend die Finanz- und Handelsflüsse in andere Länder des Südens und Ostens aufgeführt, die zwar nicht mehr Empfängerländer öffentlicher Entwicklungshilfe im Sinne des DAC seit 2005 sind, aber in früheren Aggregaten des DAC erschienen. Dabei handelt es sich um die sog. „Transitionsländer“, d.h. die mittel- und osteuropäischen Länder (MOEL) und die Neuen Unabhängigen Staaten (NUS) der früheren Sowjetunion, und um die sog „fortgeschritteneren Entwicklungsländer“. Da diese Länder keine einzelnen Kategorien mehr bilden, werden in Tabelle A.3 keine Gesamtbeträge aufgeführt. Jedoch ist interessant zu vermerken, dass der Betrag der Schweizer Ausfuhren allein nach Hongkong im Jahr 2006 den Betrag der Schweizer Ausfuhren in die Gesamtheit der am wenigsten entwickelten Länder und der einkommensschwachen Länder übertroffen hat. Zum weiteren Vergleich waren die Schweizer Exporte nach Deutschland wertmässig zehnmal höher als jene nach Hongkong. 


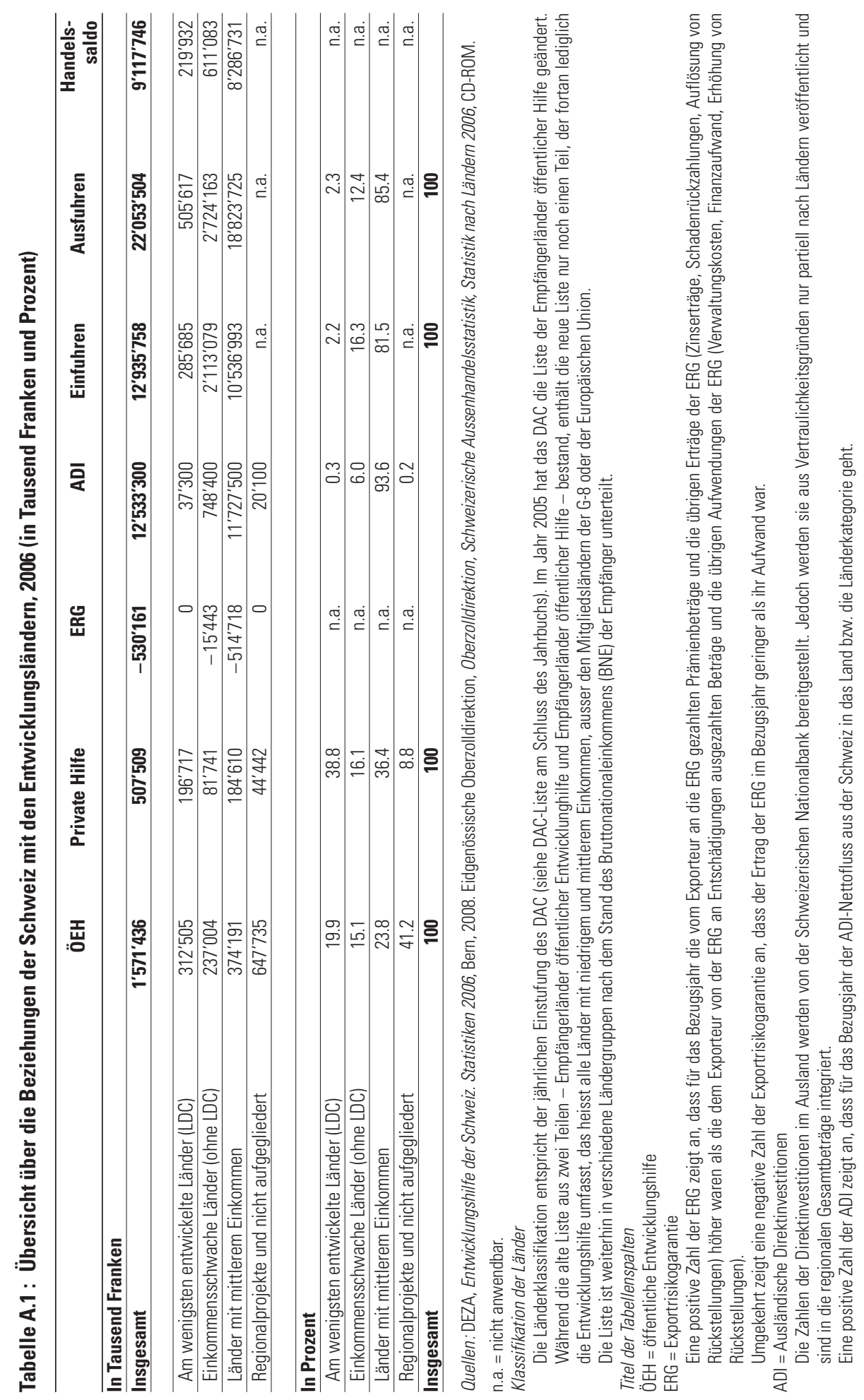




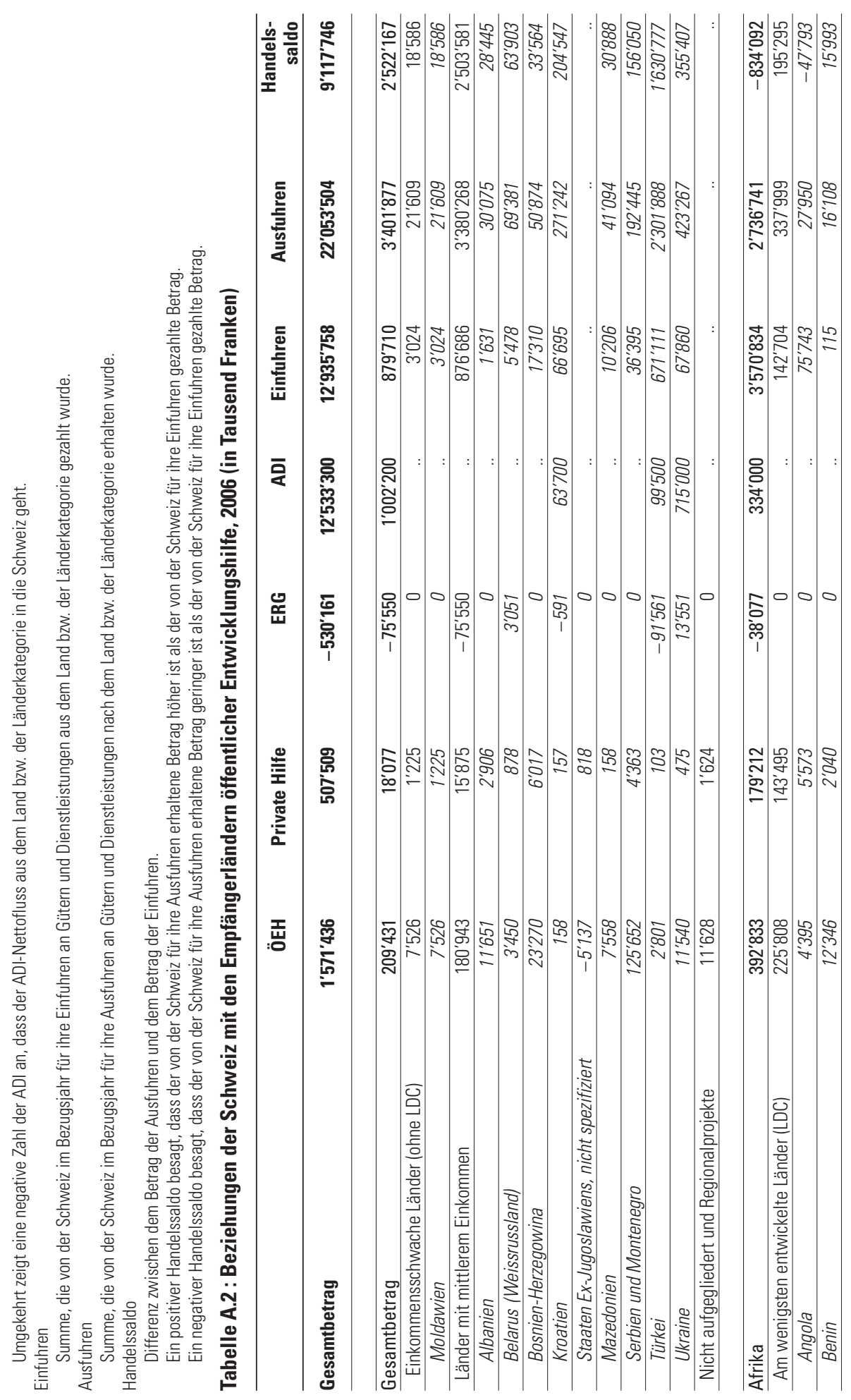




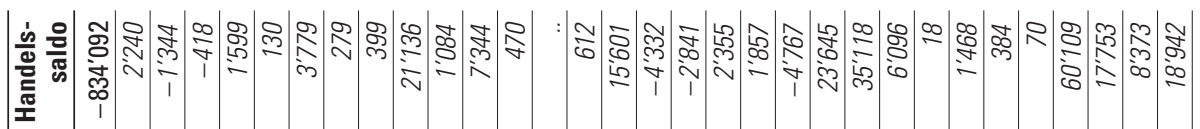

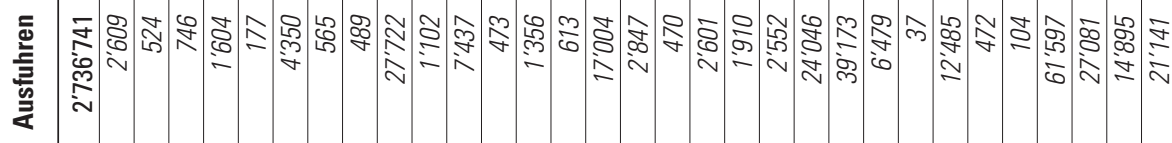

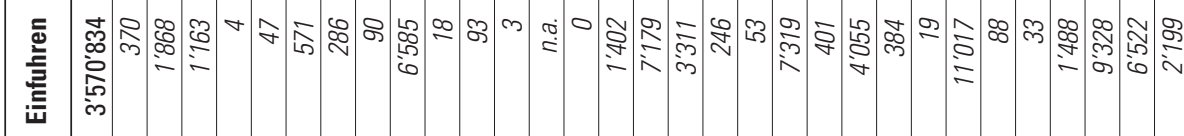

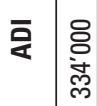

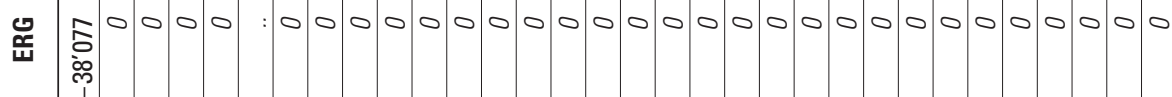

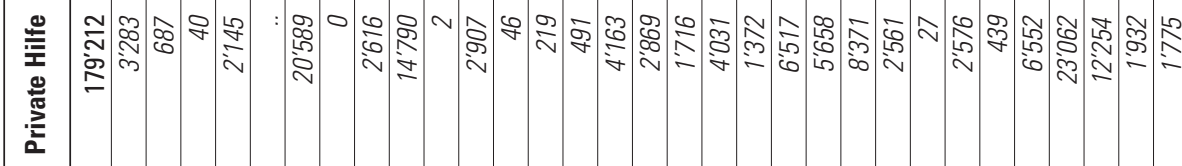

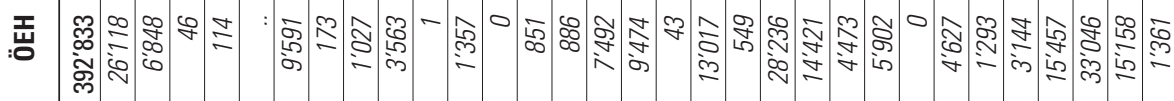

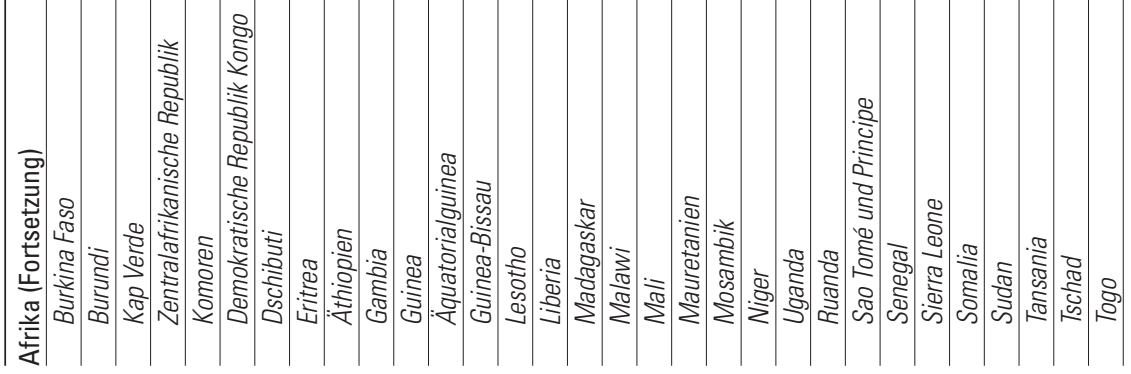




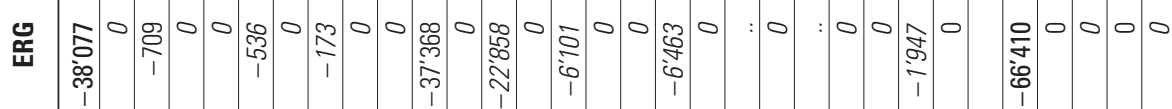

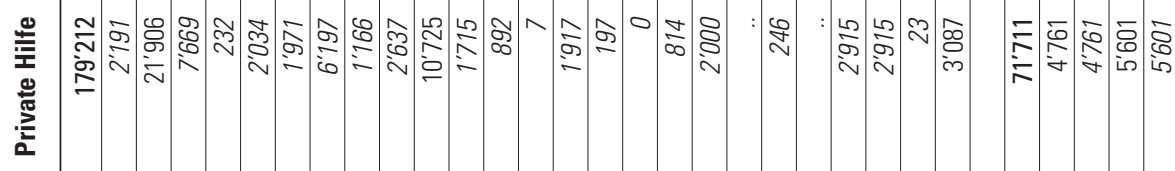

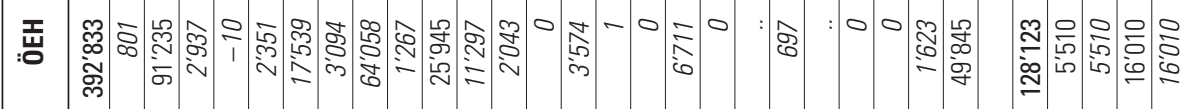

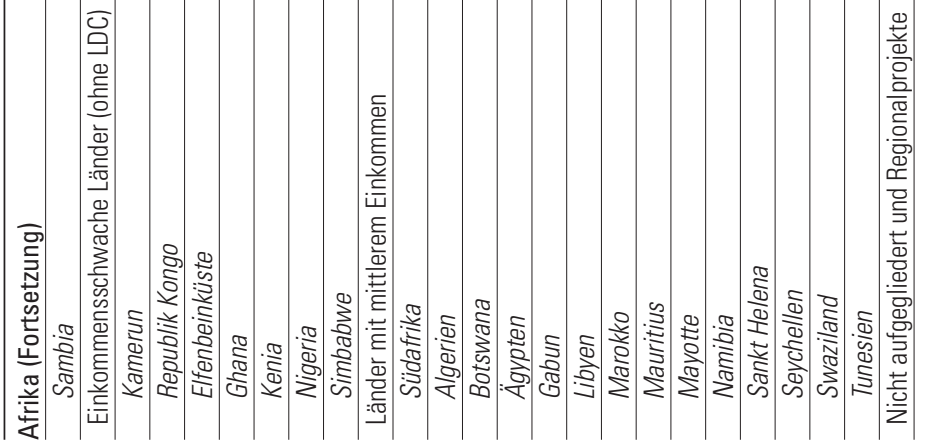

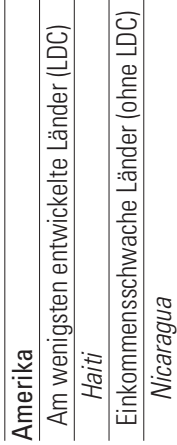




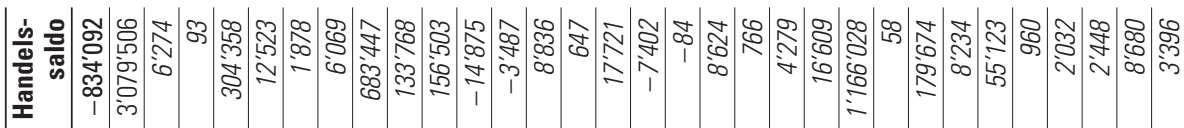

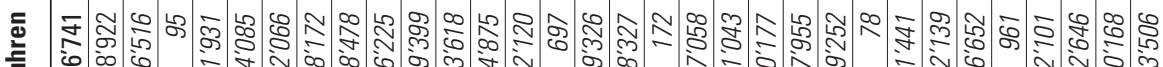

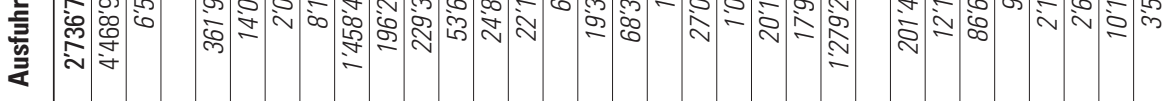

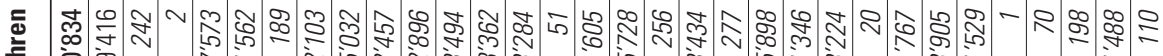

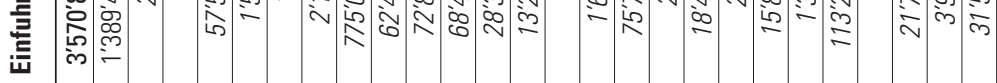

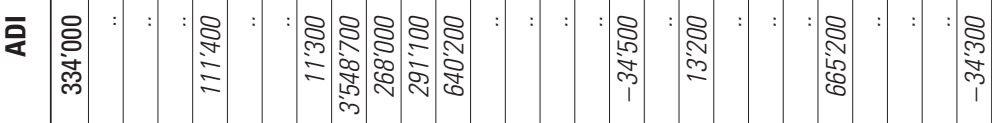

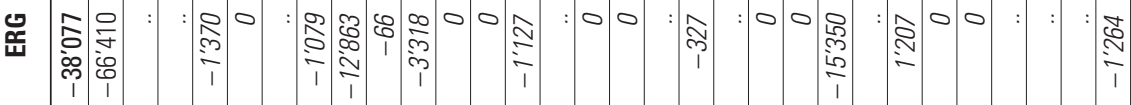

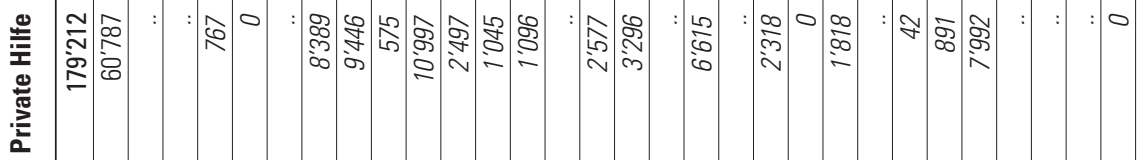

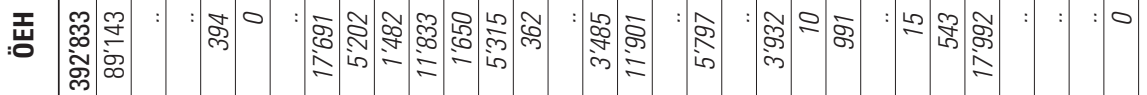

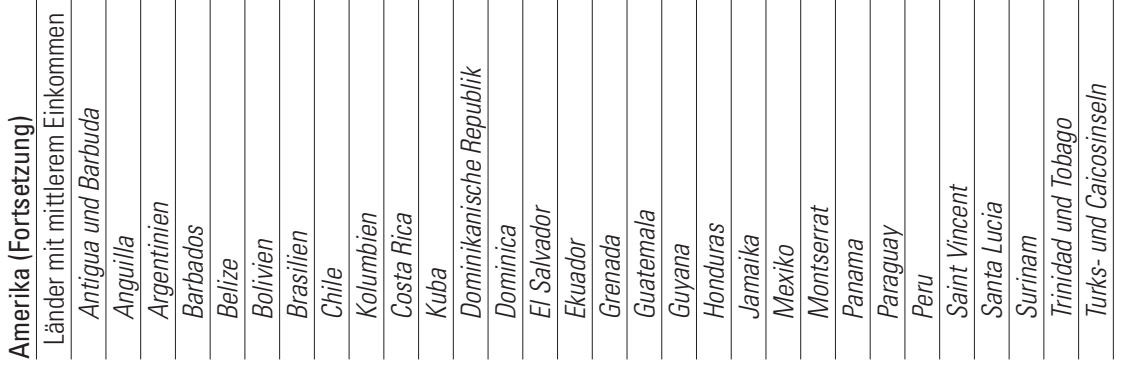




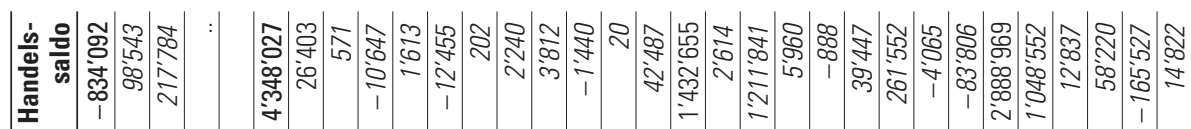

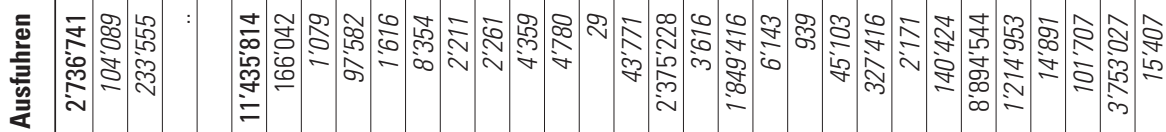

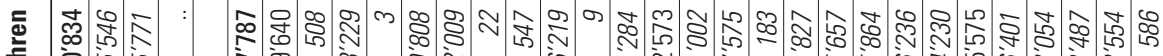

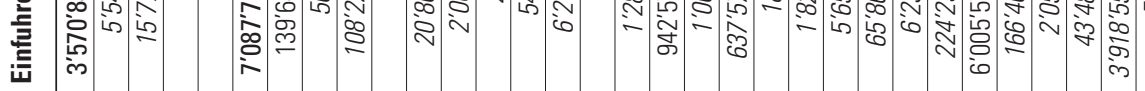

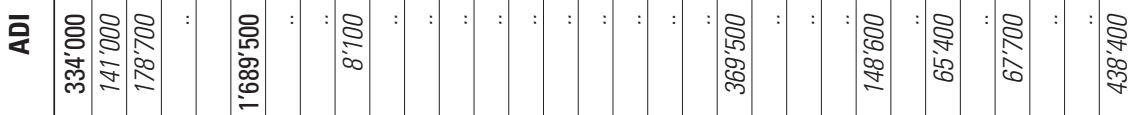

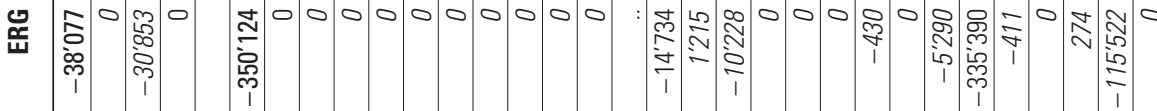

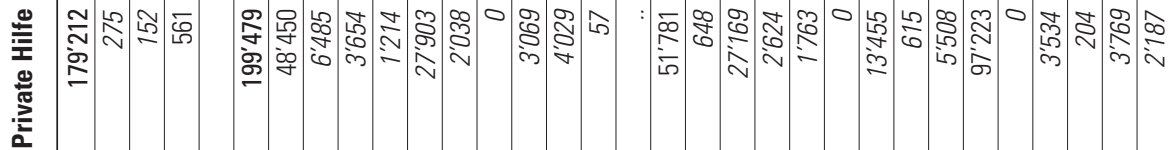

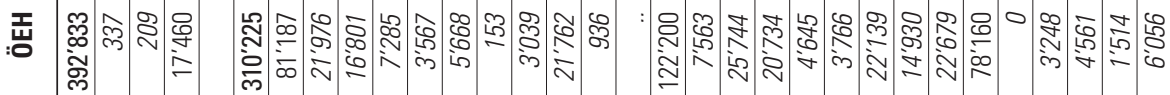

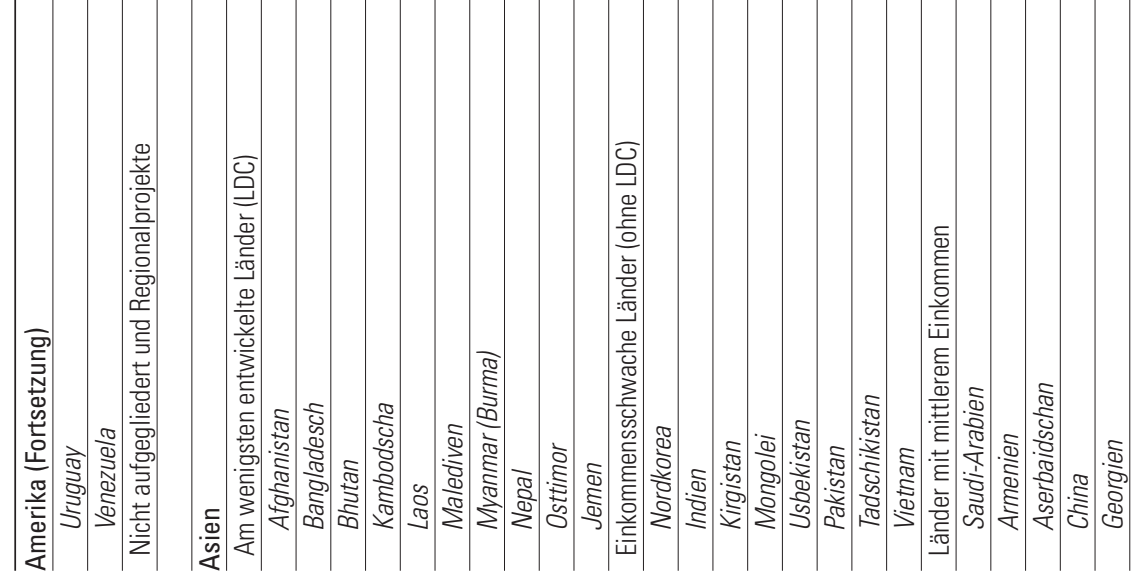




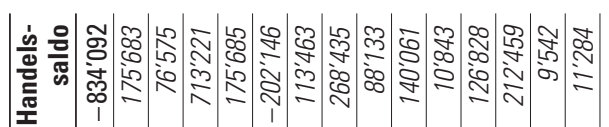

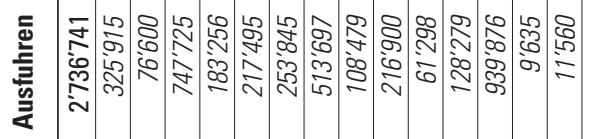

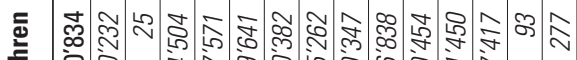

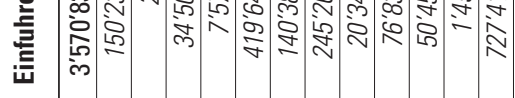

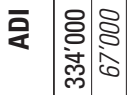

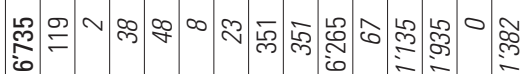

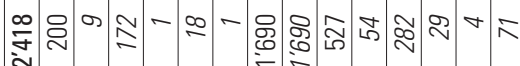

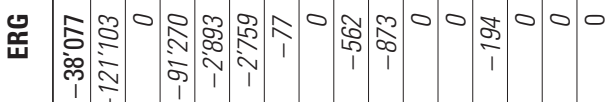

00

은

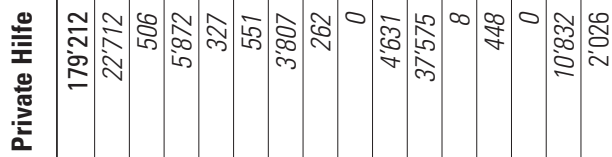

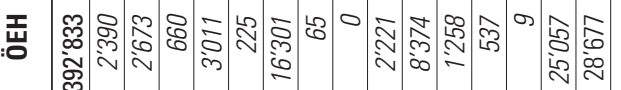

$\mathscr{g}:=$

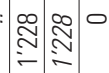

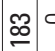

$: 0$

$: \mathbb{m}$

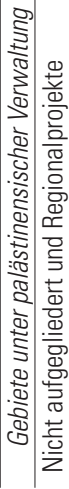

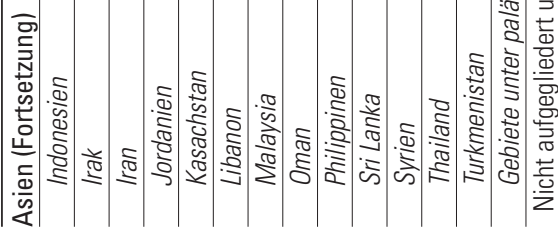

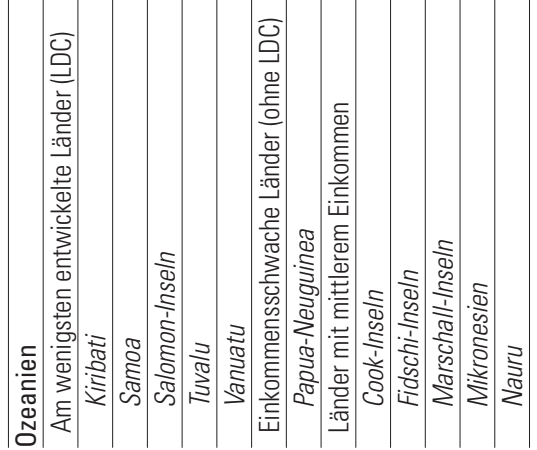




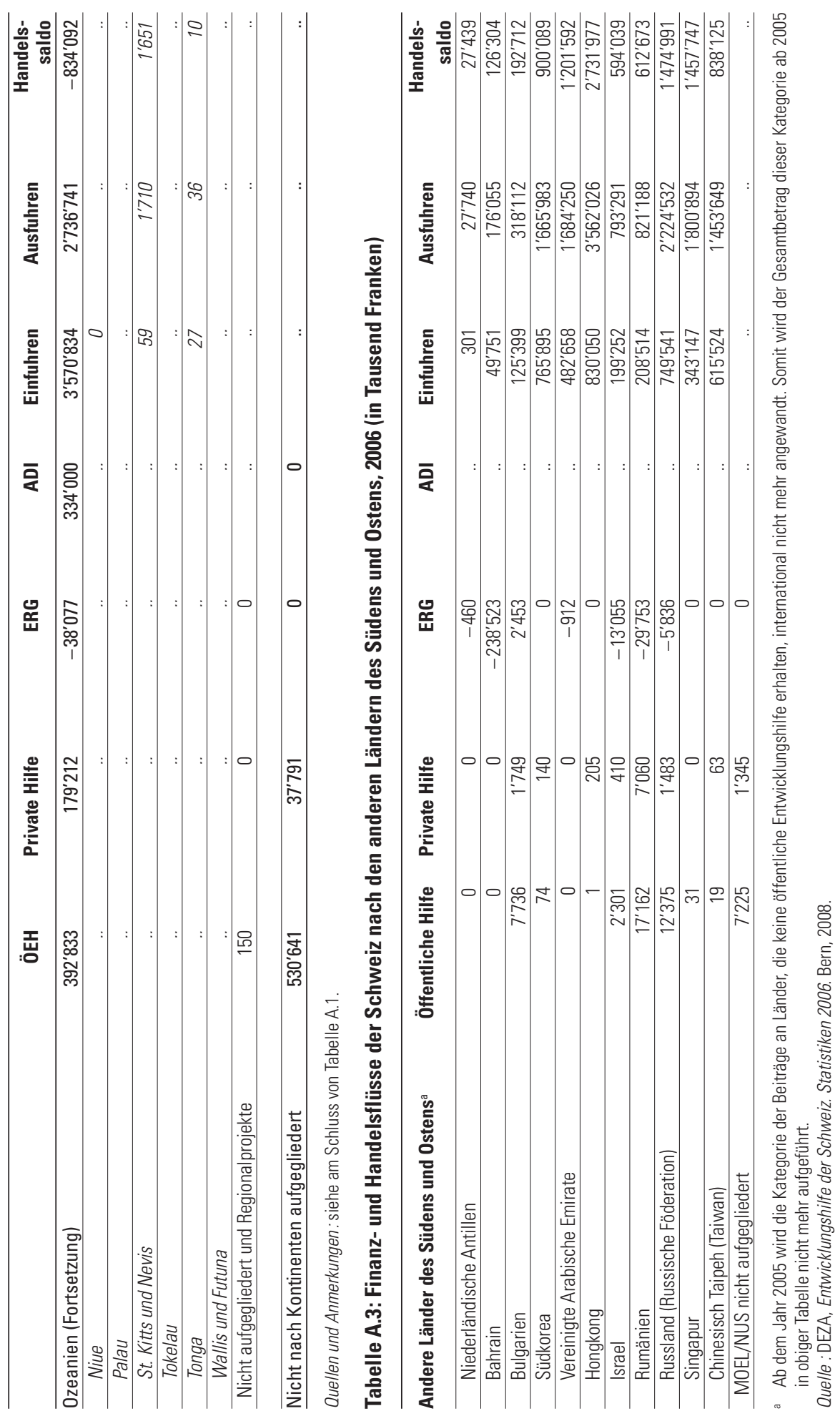

